\title{
Fixed-Time Formation Control of Multi-Robot Systems: Design and Experiments
}

DOI:

10.1109/TIE.2018.2870409

\section{Document Version}

Accepted author manuscript

Link to publication record in Manchester Research Explorer

\section{Citation for published version (APA):}

Wang, C., Tnunay, I. H. P., Zuo, Z., Lennox, B., \& Ding, Z. (2018). Fixed-Time Formation Control of Multi-Robot Systems: Design and Experiments. IEEE Transactions on Industrial Electronics.

https://doi.org/10.1109/TIE.2018.2870409

\section{Published in:}

IEEE Transactions on Industrial Electronics

\section{Citing this paper}

Please note that where the full-text provided on Manchester Research Explorer is the Author Accepted Manuscript or Proof version this may differ from the final Published version. If citing, it is advised that you check and use the publisher's definitive version.

\section{General rights}

Copyright and moral rights for the publications made accessible in the Research Explorer are retained by the authors and/or other copyright owners and it is a condition of accessing publications that users recognise and abide by the legal requirements associated with these rights.

\section{Takedown policy}

If you believe that this document breaches copyright please refer to the University of Manchester's Takedown Procedures [http://man.ac.uk/04Y6Bo] or contact uml.scholarlycommunications@manchester.ac.uk providing relevant details, so we can investigate your claim.

\section{OPEN ACCESS}




\title{
Fixed-Time Formation Control of Multi-Robot Systems: Design and Experiments
}

\author{
Chunyan Wang, Member, IEEE, Hilton Tnunay, Zongyu Zuo, Member, IEEE, Barry Lennox, Senior \\ Member, IEEE, and Zhengtao Ding, Senior Member, IEEE
}

\begin{abstract}
Time delays exist in network-connected systems. Especially for vision-based multi-robot systems, time delays are diverse and complicated due to the communication network, camera latency, image processing, etc. At the same time, many tasks, such as searching and rescue, have timing requirement. This paper focuses on fixed-time formation control of multirobot systems subject to delay constraints. First, predictorbased state transformation is employed for each robot to deal with the input delay and the uncertain terms remained in the transformed systems are carefully considered. Then, a couple of nonlinear fixed-time formation protocols are proposed for the multi-robot systems with respectively undirected and directed topology, and the corresponding settling time is derived by using the Lyapunov functions. In particular, the upper-bound estimation of the formation settling time is explicitly given irrelevant to the initial conditions. Finally, the protocols are validated through a numerical simulation example and then implemented on an E-puck robots platform. Both simulation and experimental results demonstrate the effectiveness of the proposed formation protocols.
\end{abstract}

Index Terms-Consensus control, formation control, multirobot systems, fixed-time formation, input delay.

\section{INTRODUCTION}

\section{A. Motivation and Related Work}

In recent decades, mobile robots are used to implement many tasks, such as surveillance, rural search, and exploration. As smaller, more accurate and reliable sensors and communication systems emerge, it is desirable to implement certain tasks with a group of robots rather than a single one [1], [2]. Under this background, distributed cooperative control strategies including consensus control and formation control, have attracted lots of attention and have been studied intensively in the literature, [3]-[14], to mention a few. By using distributed clustering approach, the overall computational workload in a multi-robot systems can be probabilistically reduced [15]. Furthermore, event-triggered mechanism can also be used in distributed cooperative control to reduces the unnecessary information exchange between the robots [16], [17].

C. Wang is with School of Aerospace Engineering, Beijing Institute of Technology, Beijing 100081, China (chunyan.wang@bit.edu.cn).

H. Tnunay and Z. Ding are with School of Electrical and Electronic Engineering, and Sino-British Joint Advanced Control System Technology Laboratory, University of Manchester, Sackville Street Building, Manchester M13 9PL, UK (ishak.tnunay@ postgrad.manchester.ac.uk, zhengtao.ding@manchester.ac.uk).

Z. Zuo is with the Seventh Research Division, Beihang University (BUAA), Beijing 100191, China (zzybobby@buaa.edu.cn)

B. Lennox is with School of Electrical and Electronic Engineering, The University of Manchester, M13 9PL, United Kingdom (barry.lennox@manchester.ac.uk)
Many tasks have timing requirements. For example, after a fire or tsunami disaster, the searching and rescue must be fast and efficient, and otherwise, the survival chance falls off quickly [18]. In [3], it is pointed out that the rate of convergence of multi-agent systems is related to the smallest positive eigenvalue of the graph Laplacian, which is known as algebraic connectivity of the graph. Based on the finitetime stability analysis in [19], finite-time formation protocols are designed in [20] for multi-agent systems with singleintegrator dynamics. Then, the results are extended to secondorder multi-agent systems in [21], [22]. Finite-time formation control problem for a group of nonholonomic mobile robots is considered in [23] under the leader-follower structure. Finitetime formation control of multiple second-order agents via dynamic output feedback is investigated in [24]. In the aforementioned results, the convergence time is explicitly related to the initial condition of the agents in the group. However, in many circumstances, the knowledge of initial conditions is unknown. In such a situation, the convergence time may not be estimated or designed. To overcome this problem, a class of fixed-time consensus protocols are developed in [25] and [26] for single integrator type multi-agent networks, which achieve the guaranteed upper-bound of the settling time independent of the initial conditions. The results are further extended to solve the fixed-time consensus tracking problem for doubleintegrator multi-agent systems in [27]. It is, however, worth mentioning that there is still no such a result in formation control.

With the deepening research on multi-robot systems, largescale multi-robot systems with hundreds of robots together are being proposed. Time delays arising from robots are diverse and can not be ignored. Communication delay is common for network-connected systems due to the interaction from one agent to another [28]. Another source of delay is input delay due to the decision-making and signal processing. For visionbased robots, camera latency and image processing cause delays as well [29]. There are many works focusing on multirobot systems with time delay. Formation control of a group of VTOL UAVs with certain communication delays is addressed in [30]. Time-varying formation control for a class of highorder linear multi-agent systems with time delays is studied in [31]. Frequency-domain technique is employed in [29] to deal with communication delays and the proposed results are tested with a three-robot platform. Since the consensus inputs depend on the local neighbour-to-neighbour interaction broadcast via the network, communication delays have a big impact on the input channel. Predictor-based control methods are highly used 
to deal with input delay [32] and predictor-based consensus controllers are designed in [33]-[34] for multi-agent systems with identical input delays. However, for multi-robot systems, the heterogeneity of delays for each robot prevents the use of the protocols designed in [29], [30], [33]-[34]. There is still a lack of study on formation control for multi-agent systems with non-identical input delay.

\section{B. Main Contributions}

This paper focuses on fixed-time formation control of a group of robots with delay constraints. To be specific, the contributions can be listed as follows:

First, unlike the results in [25] and [26] where only consensus protocols with undirected topologies are considered, in this paper, the results are extended to fixed-time formation control with directed topologies. The problem under consideration is more challenging and complex than that in [25] and [26].

Second, as an improvement over the existing literature in [20]-[24], the formation settling time can be controlled and designed regardless of initial conditions. Our method offers a feasible solution for some tasks which have timing requirement but the knowledge of initial conditions cannot be exactly measured.

Third, in a robotic platform, inherent time delay may exists in the input due to camera latency, image processing, decisionmaking process, and network communication. If the delay is not considered, the performance of the closed-loop systems will be degraded. Furthermore, due to the external disturbance or some other reasons, the delay time for each robot may be non-identical. This paper studies the delay effects in formation control. Compared with the results in [20]-[24] where delay is not considered and the results in [29]-[31] with identical delays, non-identical delays among the agents are considered in this paper, which is more challenging and relevant in practical view.

Finally, a multi-robot hardware platform is built and the effectiveness of the design is verified. In particular, the perfermance of static formation and time-varying formation are both provided and compared.

The remainder of this paper is organized as follows. In Section II, the problem formulation and some preliminary results are introduced. Section III presents the main results on the algorithm design and the stability analysis. Simulation and experimental results are given in Sections IV and V. Section VI concludes the paper.

\section{Problem Formulation AND PREliminaries}

\section{A. Problem Formulation}

Consider a group of $N$ mobile robots with single-integrator dynamics:

$$
\dot{x}_{i}(t)=u_{i}\left(t-h_{i}\right),
$$

where for agent $i, i=1,2, \ldots, N, x_{i} \in \mathbb{R}^{p}$ is the state, $u_{i} \in \mathbb{R}^{p}$ is the control input, $h_{i}$ is the delay time. For the convenience of presentation, in this paper, it is assumed that $p=1$. In practice, the delay time for each robot may be

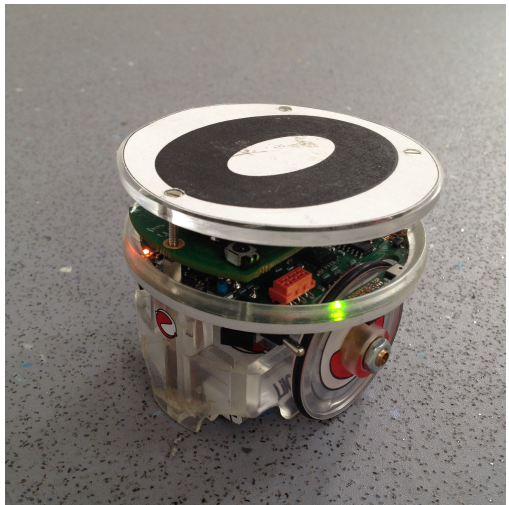

Fig. 1: E-puck robot.

slightly different due to disturbances or unstable connection. Thus, we define $h_{i}$ in a more standard form $h_{i}=h+\Delta h_{i}$.

Assumption 1: $h$ is a know constant and $\Delta h_{i}$ is unknown and bounded by $\left\|\Delta h_{i}\right\| \leq R_{1}$.

Remark 1: In this paper, we consider that the delay consists of an identical nominal part $h$ perturbed by a non-identical uncertain part $\Delta h_{i}$ which can be constant or time-varying. We will use the robust control technique to deal with this uncertainty. The only requirement for $\Delta h_{i}$ is that it is bounded by a known constant. However, the distribution of $\Delta h_{i}$ is not needed to be known.

Assumption 2: The second derivative of the state $x_{i}(t)$ is bounded, i.e., $\left|\ddot{x}_{i}(t)\right| \leq R_{2}$, where $R_{2}$ is a constant.

Remark 2: For a real system, e.g., a differential robot, $x$ represents the position and $\ddot{x}$ represents the acceleration. Assumption 2 is reasonable in the sense that the acceleration is bounded due to the physical limitation of the agent. For the Epuck robot used in our test (Fig. 1), the maximum acceleration is set to $2.5 \mathrm{~cm} / \mathrm{s}^{2}$.

Let $\mathcal{F}(t)=\left[\mathcal{F}_{1}(t), \mathcal{F}_{2}(t), \cdots, \mathcal{F}_{N}(t)\right]^{T}$ describe a timevarying formation structure of the agent network in a reference coordinate frame. The objective of this paper is to design a fixed-time formation protocol for each agent such that for any initial condition $x_{i}(0), i=1,2, \cdots, N$, there exists a formation settling time $T\left(x_{0}\right) \in[0, \infty)$ satisfying

$$
\left\{\begin{array}{l}
\lim _{t \rightarrow T\left(x_{0}\right)}\left|\left(x_{i}(t)-\mathcal{F}_{i}(t)\right)-\left(x_{j}(t)-\mathcal{F}_{j}(t)\right)\right| \rightarrow 0, \\
x_{i}(t)-x_{j}(t)=\mathcal{F}_{i}(t)-\mathcal{F}_{j}(t), \quad \forall t \geq T\left(x_{0}\right) .
\end{array}\right.
$$

Assumption 3: The specified formation vector $\mathcal{F}(t)=$ $\left[\mathcal{F}_{1}^{T}(t), \mathcal{F}_{2}^{T}(t), \cdots, \mathcal{F}_{N}^{T}(t)\right]^{T}$ is bounded with $\mathcal{F}_{i}(t), \forall i=$ $1,2, \cdots, N$ continuously differentiable, i.e., $\|\mathcal{F}(t)\| \leq c_{0}$, where $c_{0}$ is a positive constant.

\section{B. Graph Theory}

The communication connections among agents are described by a graph $\mathcal{G}(\mathcal{V}, \mathcal{E})$, where $\mathcal{V}$ represents the agents and $\mathcal{E}$ represents the connections between the agents. In the directed graph, $(i, j) \in \mathcal{E}$ represents the communication from the $i$ th agent to the $j$ th agent, but not vice versa. In the undirected graph, $(i, j) \in \mathcal{E}$ means that the information can be exchanged between the $i$ th agent and $j$ th agent. For $N$ 
agents, the associated adjacency matrix of $\mathcal{G}$ is defined as $A=\left[a_{i j}\right]_{N \times N} \in \mathbb{R}^{N \times N}$. If there is a connection from agent $j$ to agent $i, a_{i j}=1$; otherwise $a_{i j}=0$. The Laplacian matrix $\mathcal{L}=\left[l_{i j}\right]_{N \times N}$ associated with $A$ is defined by $l_{i i}=\sum_{j=1}^{N} a_{i j}$ and $l_{i j}=-a_{i j}$ when $i \neq j$. For an undirected graph $\mathcal{G}$, if it is connected, the Laplacian matrix $\mathcal{L}$ has a single zero eigenvalue with $\mathcal{L} \mathbf{1}_{N}=0$, and all the other eigenvalues of $\mathcal{L}$ are real and positive. Furthermore, we have the following properties for the Laplacian matrix $\mathcal{L}$ from [4]:

1) $x^{T} \mathcal{L} x=\frac{1}{2} \sum_{i=1}^{N} \sum_{j=1}^{N} a_{i j}\left(x_{j}-x_{i}\right)^{2}$;

2) $\lambda_{2}(\mathcal{L})=\min _{x \neq 0,1_{N}^{T} x=0} \frac{x^{T} \mathcal{L} x}{x^{T} x}$

3) $x^{T} \mathcal{L} x \geq \lambda_{2}(\mathcal{L}) x^{T} x$, where $\lambda_{2}(\mathcal{L})$ is the smallest positive eigenvalue of $\mathcal{L}$.

\section{Preliminaries}

Lemma 1: [19] For a system $\dot{\chi}(t)=h(\chi, t), \chi(0)=\chi_{0}$, in which $h(\chi): \mathbb{R}^{n} \rightarrow \mathbb{R}^{n}$ is continuous and $h(0)=0$. If $V(\chi): \mathbb{R}^{n} \rightarrow \mathbb{R}$ is a continuously differentiable positive definite function and there exist real numbers $\varepsilon>0, \lambda \in$ $(0,1)$ such that $\dot{V}(\chi)+\varepsilon V^{\lambda}(\chi) \leq 0$, then, the origin is a globally finite-time stable equilibrium of the system and the settling time is $T\left(\chi_{0}\right) \leq \frac{1}{\varepsilon(1-\lambda)} V^{(1-\lambda)}\left(\chi_{0}\right)$.

Lemma 2: [35] For a scalar system $\dot{\chi}(t)=\mathfrak{u}(t), \chi(0)=0$, if the controller is chosen as $\mathfrak{u}(t)=-\alpha \chi^{2-\frac{m}{n}}(t)-\beta \chi^{\frac{m}{n}}(t)$ with $\alpha, \beta>0$ and $m, n$ are both positive odd integers satisfying $m<n$, the closed-loop system is fixed-time stable at the origin with globally bounded settling time $T\left(\chi_{0}\right) \leq$ $\frac{n \pi}{2 \sqrt{\alpha \beta(n-m)}}$.

Lemma 3: [25] Let $\beta_{1}, \beta_{2}, \cdots, \beta_{N} \geq 0$ and $0<q<1$. Then $\sum_{j=1}^{N} \beta_{j}^{q} \geq\left(\sum_{j=1}^{N} \beta_{j}\right)^{q}$.

Lemma 4: (Young's Inequality) For nonnegative real numbers $a, b$, if $p, q$ are real numbers that satisfy $\frac{1}{p}+\frac{1}{q}=1$, then $a b \leq \frac{a^{p}}{p}+\frac{b^{q}}{q}$.

\section{Algorithms Design}

\section{A. Fixed-Time Formation with Undirected Topology}

To deal with the input delay, one basic idea is to predict the evolution of state variable for the delay period and then use the predicted state for control [32], [33]. For multi-robot systems (1), we propose a new state $y(t)$ to predict the state $x$ at time $t+h$ :

$$
y_{i}(t)=x_{i}(t)-\mathcal{F}_{i}(t)+\int_{t}^{t+h}\left[u_{i}(\tau-h)-\dot{\mathcal{F}}_{i}(\tau)\right] \mathrm{d} \tau .
$$

Differentiating $y_{i}(t)$ against time yields

$$
\begin{aligned}
\dot{y}_{i}(t)= & u_{i}\left(t-h-\Delta h_{i}\right)-\dot{\mathcal{F}}_{i}(t)+u_{i}(t)-u_{i}(t-h) \\
& -\dot{\mathcal{F}}_{i}(t+h)+\dot{\mathcal{F}}_{i}(t) \\
= & u_{i}(t)+\tilde{u}_{i}(t)-\dot{\mathcal{F}}_{i}(t+h),
\end{aligned}
$$

where $\tilde{u}_{i}(t)=u_{i}\left(t-h-\Delta h_{i}\right)-u_{i}(t-h)$, which represents the uncertain term remains in the transformed system. Before we start the fixed-time formation analysis, a result on the bound of $\tilde{u}_{i}(t)$ is given first.

Lemma 5: If Assumptions 1 and 2 hold, the uncertain term $\tilde{u}_{i}(t)$ in the transformed system (4) is bounded and a bound can be established as $0 \leq\left|\tilde{u}_{i}(t)\right| \leq R_{1} R_{2}$.
Proof: From system (1), we have $\tilde{u}_{i}(t)=\dot{x}_{i}(t)-\dot{x}_{i}(t+$ $\left.\Delta h_{i}\right)=-\int_{t}^{t+\Delta h_{i}} \ddot{x}(\tau) \mathrm{d} \tau$ and $\left|\tilde{u}_{i}(t)\right|=\left|\int_{t}^{t+\Delta h_{i}} \ddot{x}(\tau) \mathrm{d} \tau\right| \leq$ $\int_{t}^{t+R_{1}} R_{2} \mathrm{~d} \tau \leq R_{1} R_{2}$, for all $t \in[0,+\infty)$, which completes the proof.

The signal collected by agent $i$ about the information of its neighbouring agents is given by

$$
\zeta_{i}(t)=\sum_{j=1}^{N} a_{i j}\left(y_{i}(t)-y_{j}(t)\right), i=1,2, \cdots, N .
$$

Based on the above preparation, now we present a nonlinear formation protocol for each agent:

$$
u_{i}(t)=-\alpha \zeta_{i}^{2-\frac{m}{n}}(t)-\beta \zeta_{i}^{\frac{m}{n}}(t)-\gamma \operatorname{sgn}\left(\zeta_{i}(t)\right)+\dot{\mathcal{F}}_{i}(t+h),
$$

where $\alpha, \beta, m, n>0$ are a set of design parameters, $\gamma$ is a number satisfying $\gamma \geq R_{1} R_{2}, \operatorname{sgn}(\cdot)$ denotes the signum function. In particular, we have the following assumption.

Assumption 4: $m, n$ are both positive odd integers satisfying $m<n$.

Theorem 1: For input-delayed multi-robot systems (1) with the associated undirected graph $\mathcal{G}$ connected, the fixed-time formation problem can be solved by controller (5). Furthermore, the settling time satisfies

$$
T\left(x_{0}\right) \leq T_{\max }:=\frac{\pi n N^{\frac{n-m}{4 n}}}{2 \sqrt{\alpha \beta} \lambda_{2}(\mathcal{L})(n-m)}+h .
$$

Proof: Putting (4) and (5) together, we obtain $\dot{y}_{i}(t)=$ $-\alpha\left(\zeta_{i}(t)\right)^{2-\frac{m}{n}}-\beta\left(\zeta_{i}(t)\right)^{\frac{m}{n}}+\tilde{u}_{i}(t)-\gamma \operatorname{sgn}\left(\zeta_{i}(t)\right)$. Let $y(t)=$ $\left[y_{1}(t), y_{2}(t), \cdots, y_{N}(t)\right]^{T}$ and consider the following semipositive definite function:

$$
V(y(t))=\frac{1}{2} y^{T} \mathcal{L} y=\frac{1}{4} \sum_{i=1}^{N} \sum_{j=1}^{N} a_{i j}\left(y_{i}(t)-y_{j}(t)\right)^{2} .
$$

Since $\mathcal{G}$ is connected, zero is a simple eigenvalue of $\mathcal{L}$, which implies that $V(y(t))=0$ if and only if $y(t) \in \operatorname{span}\left\{\mathbf{1}_{N}\right\}$ which implies that $\lim _{t \rightarrow \infty}\left(y_{i}(t)-y_{j}(t)\right)=0$. From (3) and (5), it is concluded that $\lim _{t \rightarrow \infty}\left(y_{i}(t)-y_{j}(t)\right)=0 \Longrightarrow$ $\lim _{t \rightarrow \infty}\left(x_{i}(t)-x_{j}(t)\right)=\mathcal{F}_{i}(t)-{ }^{t \rightarrow \infty} \mathcal{F}_{j}(t), \forall i, j \in N$. Differentiating $V(y)$ against time yields

$$
\begin{aligned}
\dot{V}(y)= & \sum_{i=1}^{N} \frac{\partial V(y)}{\partial y_{i}} \dot{y}_{i}(t) \\
= & -\alpha \sum_{i=1}^{N} \zeta_{i}^{\frac{3 n-m}{n}}(t)-\beta \sum_{i=1}^{N} \zeta_{i}^{\frac{m+n}{n}}(t) \\
& -\gamma \sum_{i=1}^{N}\left|\zeta_{i}(t)\right|+\sum_{i=1}^{N} \tilde{u}_{i}(t)\left(\zeta_{i}(t)\right) \\
\leq & -\alpha \sum_{i=1}^{N}\left(\zeta_{i}^{2}(t)\right)^{\frac{3 n-m}{2 n}}-\beta \sum_{i=1}^{N}\left(\zeta_{i}^{2}(t)\right)^{\frac{m+n}{2 n}} \\
& -\left(\gamma-R_{1} R_{2}\right) \sum_{i=1}^{N}\left|\zeta_{i}(t)\right|
\end{aligned}
$$

where $\frac{\partial V(y)}{\partial y_{i}}=\sum_{j=1}^{N} a_{i j}\left(y_{i}(t)-y_{j}(t)\right)$ due to the symmetry of $\mathcal{A}$ is inserted to derive the second equality. 
With Lemmas 3 and 5, we have

$$
\begin{aligned}
\dot{V}(y) \leq & -\alpha N^{\frac{n-m}{2 n}}\left(\sum_{i=1}^{N}\left(\sum_{j=1}^{N} a_{i j}\left(y_{i}(t)-y_{j}(t)\right)\right)^{2}\right)^{\frac{3 n-m}{2 n}} \\
& -\beta\left(\sum_{i=1}^{N}\left(\sum_{j=1}^{N} a_{i j}\left(y_{i}(t)-y_{j}(t)\right)\right)^{2}\right)^{\frac{m+n}{2 n}}
\end{aligned}
$$

Since $\mathcal{L}$ is semi-positive definite, we can obtain a matrix $H \in \mathbb{R}^{N \times N}$ such that $\mathcal{L}=H^{T} H$, and thus

$$
\begin{aligned}
& \frac{\sum_{i=1}^{N}\left(\sum_{j=1}^{N} a_{i j}\left(y_{i}(t)-y_{j}(t)\right)\right)^{2}}{V(y)}=\frac{y^{T} \mathcal{L}^{T} \mathcal{L} y}{\frac{1}{2} y^{T} \mathcal{L} y} \\
& =\frac{2 y^{T} H^{T} H H^{T} H y}{y^{T} H^{T} H y}=\frac{2 y^{T} H^{T} \mathcal{L}^{T} H y}{y^{T} H^{T} H y} \geq 2 \lambda_{2}(\mathcal{L}) .
\end{aligned}
$$

Putting (8) and (9) together, we have

$$
\begin{aligned}
\dot{V}(y) & \leq-\alpha N^{\frac{n-m}{2 n}}\left(2 \lambda_{2}(\mathcal{L}) V\right)^{\frac{3 n-m}{2 n}}-\beta\left(2 \lambda_{2}(\mathcal{L}) V\right)^{\frac{m+n}{2 n}} \\
& =-\left(\alpha N^{\frac{n-m}{2 n}}\left(2 \lambda_{2}(\mathcal{L}) V\right)^{\frac{n-m}{n}}+\beta\right)\left(2 \lambda_{2}(\mathcal{L}) V\right)^{\frac{m+n}{2 n}} .
\end{aligned}
$$

If $V(y) \neq 0$, we define $\xi=\sqrt{2 \lambda_{2}(\mathcal{L}) V(y)}$. Then, we obtain $\dot{\xi}=\lambda_{2}(\mathcal{L}) \dot{V}(y) / \sqrt{2 \lambda_{2}(\mathcal{L}) V(y)}$ and $\dot{\xi}=$ $-\alpha N^{\frac{n-m}{2 n}} \lambda_{2}(\mathcal{L}) \xi^{\frac{2 n-m}{n}}-\beta \lambda_{2}(\mathcal{L}) \xi^{\frac{m}{n}}$. Based on Lemma 2, we have $\lim _{t \rightarrow T\left(y_{0}\right)} V(y)=0$ with the settling time bounded by

$$
\begin{aligned}
T\left(y_{0}\right) & =\frac{n N^{\frac{n-m}{4 n}}}{\sqrt{\alpha \beta} \lambda_{2}(\mathcal{L})(n-m)} \tan ^{-1}\left(N^{\frac{n-m}{4 n}} \sqrt{\frac{\alpha}{\beta}} V(y(0))\right) \\
& \leq \frac{\pi n N^{\frac{n-m}{4 n}}}{2 \sqrt{\alpha \beta} \lambda_{2}(\mathcal{L})(n-m)} .
\end{aligned}
$$

The fixed-time consensus of the transformed system (4) is achieved.

From (10), we know that $y(t) \in \operatorname{span}\left\{\mathbf{1}_{N}\right\}$ within fixed time $\frac{\pi n N \frac{n-m}{4 n}}{2 \sqrt{\alpha \beta} \lambda_{2}(\mathcal{L})(n-m)}$. Therefore, we have $x_{i}(t)-\mathcal{F}_{i}(t)=$ $y_{i}(t)-\int_{t}^{t+h}\left[u_{i}(\tau-h)-\dot{\mathcal{F}}_{i}(\tau)\right] \mathrm{d} \tau$ and $u_{i}(t)=\dot{\mathcal{F}}_{i}(t+h)$ for all $t \geq \frac{\pi n N^{\frac{n-m}{4 n}}}{2 \sqrt{\alpha \beta} \lambda_{2}(\mathcal{L})(n-m)}$. It is derived that $x_{i}(t)-\mathcal{F}_{i}(t)=y_{i}(t)$ for all $t \geq \frac{\pi n N^{\frac{n-m}{4 n}}}{2 \sqrt{\alpha \beta} \lambda_{2}(\mathcal{L})(n-m)}+h$. Thus, we have $T\left(x_{0}\right) \leq$ $\frac{\pi n N^{\frac{n-m}{4 n}}}{2 \sqrt{\alpha \beta} \lambda_{2}(\mathcal{L})(n-m)}+h$, and

$$
\left\{\begin{array}{l}
\lim _{t \rightarrow T(x)}\left|\left(x_{i}(t)-\mathcal{F}_{i}(t)\right)-\left(x_{j}(t)-\mathcal{F}_{j}(t)\right)\right| \rightarrow 0, \\
x_{i}(t)-x_{j}(t)=\mathcal{F}_{i}(t)-\mathcal{F}_{j}(t), \forall t \geq T(x),
\end{array}\right.
$$

which completes the proof.

Remark 3: The estimation for settling time $T\left(x_{0}\right)$ is only related to the delay $h$, the design parameters $m$ and $n$, the algebraic connectivity $\lambda_{2}(\mathcal{L})$ of the graph Laplacian, and the group order $N$. Therefore, the upper-bound $T_{\max }$ of the formation settling time can be designed independent of the initial conditions.

\section{B. Fixed-Time Formation with directed topology}

In the previous subsection, a control protocol for fixed-time formation is developed under undirected communication. To reduce the communication burden and facilitate the practical applications, in this subsection, we will develop a fixedtime formation protocol based on directed local neighbourto-neighbour interaction.

Assumption 5: The directed graph $\mathcal{G}(A)$ is strongly connected.

Lemma 6: [2] If $\mathcal{G}(A)$ is strongly connected, then $\mathcal{L}$ has a simple eigenvalue at zero and there exists a positive vector $\delta=\left[\delta_{1}, \delta_{2}, \cdots, \delta_{N}\right]^{T} \in \mathbb{R}^{N}$ such that $\delta^{T} \mathcal{L}=0$.

Definition 1: [3] Let $\mathcal{G}(A)=\{\mathcal{V}, \mathcal{E}, A\}$ be weighted digraph. Let $\tilde{\mathcal{E}}$ be the set of reverse edges of $\mathcal{G}(A)$ obtained by reversing the order of nodes of all pair in $\mathcal{E}$. The mirror of $\mathcal{G}(A)$ denoted by $\mathcal{G}(\hat{A})=\{\mathcal{V}, \hat{\mathcal{E}}, \hat{A}\}$ with the same set of nodes as $\mathcal{G}(A)$, the set of edges $\hat{\mathcal{E}}=\mathcal{E} \cup \tilde{\mathcal{E}}$, and the symmetric adjacent matrix $\hat{A}=\left[\hat{a}_{i j}\right]_{N \times N}$ with elements $\hat{a}_{i j}=\hat{a}_{j i}=\left(\delta_{i} a_{i j}+\delta_{j} a_{j i}\right) / 2$.

Lemma 7: [36] Let $\mathcal{G}(A)$ be weighted digraph with Laplacian matrix $\mathcal{L}$. Then, $\hat{\mathcal{L}}=\left(\Delta \mathcal{L}+\mathcal{L}^{T} \Delta\right) / 2$ is a valid Laplacian matrix for $\mathcal{G}(\hat{A})$, where $\Delta=\operatorname{diag}\left\{\delta_{1}, \delta_{2}, \cdots, \delta_{N}\right\}$ is the diagonal matrix whose diagonal entries are given by $\delta_{i}$, $i=1,2, \cdots, N$.

The signal collected by agent $i$ about the information of its neighbouring agents is given by

$$
\hat{\zeta}_{i}(t)=\sum_{j=1}^{N} \hat{a}_{i j}\left(y_{i}(t)-y_{j}(t)\right), i=1,2, \cdots, N .
$$

A nonlinear protocol that solves the fixed-time formation problem for the multi-robot systems with directed topology can be proposed as

$$
u_{i}(t)=-\alpha \hat{\zeta}_{i}^{2-\frac{m}{n}}(t)-\beta \hat{\zeta}_{i}^{\frac{m}{n}}(t)-\gamma \operatorname{sgn}\left(\hat{\zeta}_{i}(t)\right)+\dot{\mathcal{F}}_{i}(t+h)
$$

where $\alpha, \beta, \gamma, m, n$ are defined in (5), $y_{i}$ and $y_{j}$ are given in (3), and $\hat{a}_{i j}$ is given in Definition 1.

Theorem 2: With Assumption 5, the fixed-time formation problem of multi-robot systems (1) can be solved by the algorithm (11). Furthermore, the settling time satisfies $T(x) \leq$ $\frac{\pi n N^{\frac{n-m}{4 n}}}{2 \sqrt{\alpha \beta} \lambda_{2}(\hat{\mathcal{L}})(n-m)}+h$, where $\lambda_{2}(\hat{\mathcal{L}})$ is the smallest positive eigenvalue of $\hat{\mathcal{L}}$.

Proof: The proof is similar to that of Theorem 1 and hence omitted.

Remark 4: From (2) and Assumption 3, it can be seen that the consensus of all robots can be achieved if $\mathcal{F}(t) \equiv 0$. Thus, the fixed-time consensus problem studied in [25] can be viewed as a special case of the fixed-time formation problem. If $\Delta h_{i} \equiv 0, \forall i=1,2, \cdots, N$, the delay problem under consideration is equivalent to the identical delay case considered in [31] and [33].

\section{Simulations}

This section presents some simulation results on convergence of six agents with $\mathcal{F}(t)=0$. The connection is shown in Fig. 2. In the simulation, nonzero $a_{i j}=1, \alpha=\beta=4$, $m=7$ and $n=9$ for each protocol, the time delay of the system $h=0.3, \Delta h_{i}=0.05 \sin (i \cdot t), \forall i=1,2, \cdots, 6$. The 


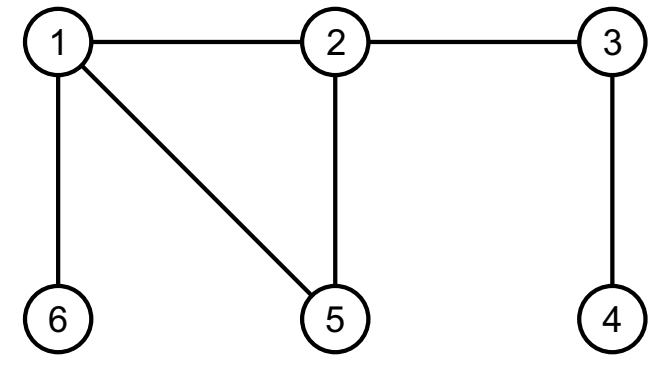

Fig. 2: Communication topology.

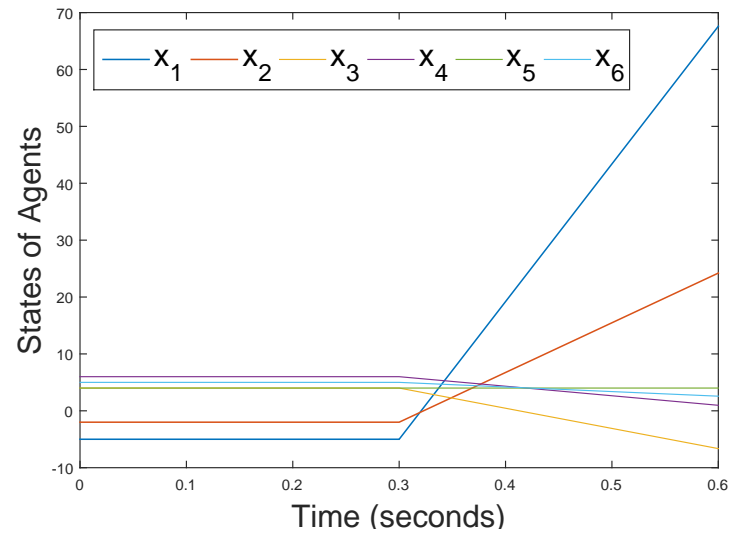

Fig. 3: State trajectories of 6 agents under the controller designed in [25] with $\Delta h_{i}=0, h=0.03$ (Case 1).

adjacency matrix $A$ is given by

$$
A=\left[\begin{array}{llllll}
0 & 1 & 0 & 0 & 1 & 1 \\
1 & 0 & 1 & 0 & 1 & 0 \\
0 & 1 & 0 & 1 & 0 & 0 \\
0 & 0 & 1 & 0 & 0 & 0 \\
1 & 1 & 0 & 0 & 0 & 0 \\
1 & 0 & 0 & 0 & 0 & 0
\end{array}\right] .
$$

The Laplacian matrix is given by

$$
\mathcal{L}=\left[\begin{array}{cccccc}
3 & -1 & 0 & 0 & -1 & -1 \\
-1 & 3 & -1 & 0 & -1 & 0 \\
0 & -1 & 2 & -1 & 0 & 0 \\
0 & 0 & -1 & 1 & 0 & 0 \\
-1 & -1 & 0 & 0 & 2 & 0 \\
-1 & 0 & 0 & 0 & 0 & 1
\end{array}\right]
$$

The eigenvalues of the Laplacian matrix $\mathcal{L}$ are $\{0,0.4131,1.1369,2.3595,3.6977,4.3928\}$, and zero is a simple eigenvalue. We obtain that $\lambda_{2}(\mathcal{L})=0.4131$. The estimated upper bound of the settling time for protocol (5) is 5.0231. Consider two initial conditions: (1) $x(0)=[10,-20,-3,9,4,-30]^{T}$ and (2) $x(0)=[100,-200,-30,90,40,-300]^{T}$.

To demonstrate the communication and input delay effect in the consensus control, first we will show the simulation results with the controllers designed in [25]. Figures 3 and 4 show that the consensus cannot be achieved by the controllers given in [25] even if the delays are identical and very small $h=0.03 s$.

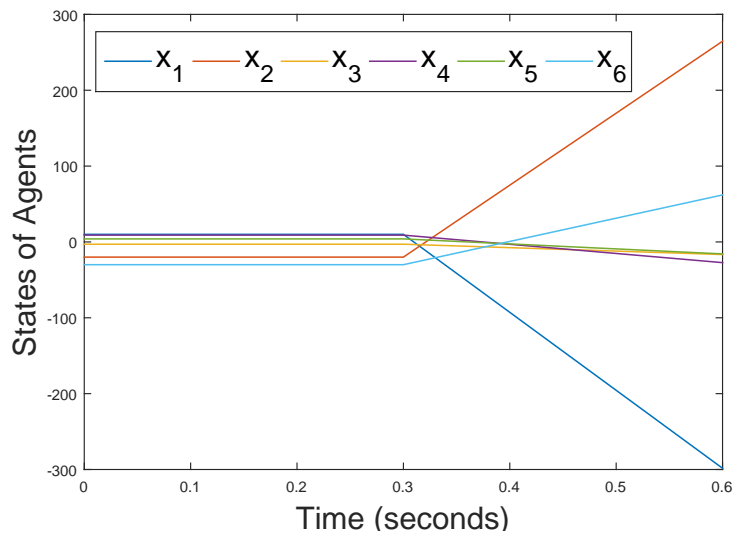

Fig. 4: State trajectories of 6 agents under the controller designed in [25] with $\Delta h_{i}=0, h=0.03$ (Case 2).

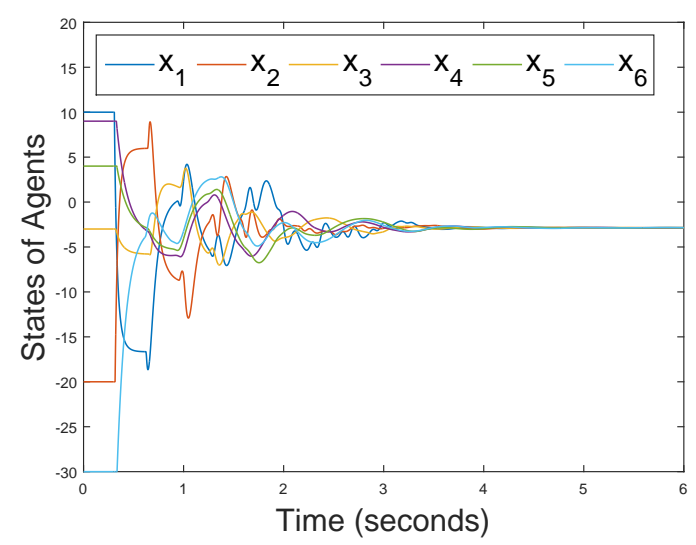

Fig. 5: State trajectories of 6 agents with $h=0.3, \Delta h_{i}=$ $\sin (i * t), i=1,2, \cdots, 6$. (Case 1).

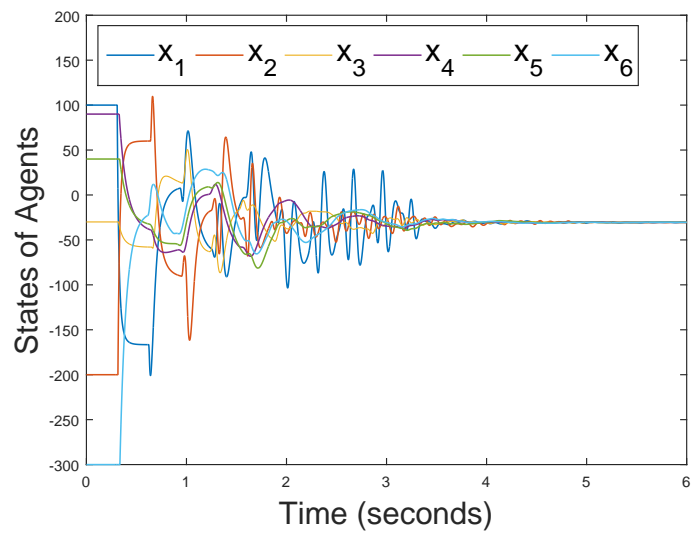

Fig. 6: State trajectories of 6 agents with $h=0.3, \Delta h_{i}=$ $\sin (i * t), i=1,2, \cdots, 6$. (Case 2). 
From the results shown in Figs 5 and 6, it is demonstrated that the convergence performance is guaranteed with the controller designed in this paper under different initial conditions. In addition, the numerical results given in Fig. 5 show that the settling time of the consensus protocol is about $5 \mathrm{~s}$, which demonstrates the correctness of the estimation derived in Theorem 1.

\section{EXPERIMENT VALIDATION}

\section{A. Experimental Platform}

An experimental testbed, see Fig. 7, is designed to demonstrate the performance of formation protocols with real robotic systems. In this testbed, a Linux-based host computer (2.7$\mathrm{GHz}$ clock speed, dual processor, 4-GB RAM, and equipped with Robot Operating Systems) receives position information of four E-puck mobile robots from a camera (Logitech C525, HD 720p). The camera used here is to localize the robots within the workspace area $(100 \mathrm{~cm} \times 100 \mathrm{~cm})$. Some static landmarks are used by the camera to calibrate the workspace coordinates. Images captured by the camera are then processed by utilizing image processing software Swarmcon [37] to provide the positions of robots. The way this software locates the robots is by tracking the unique patterns attached to them. The positions obtained are subsequently applied to the proposed control law to produce the control inputs. Finally, these inputs are transmitted over the network via Bluetooth connection.

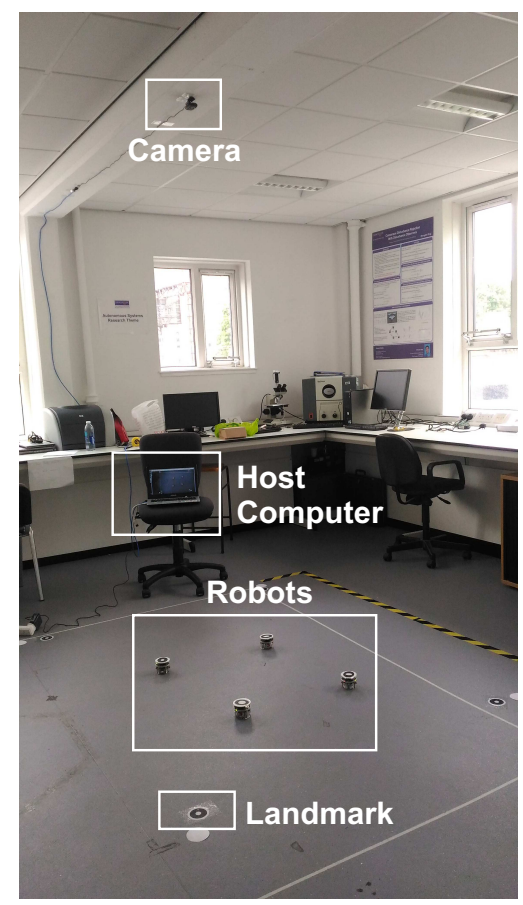

Fig. 7: Experimental Platform

In this system, an inherent time delay whose value is around $0.15 \mathrm{~s}$ to $0.195 \mathrm{~s}$ exists in the input due to camera latency, image processing, decision-making process, and Bluetooth communication [29]. Since the Bluetooth broadcast to the robots is done sequentially, the delays are in general not

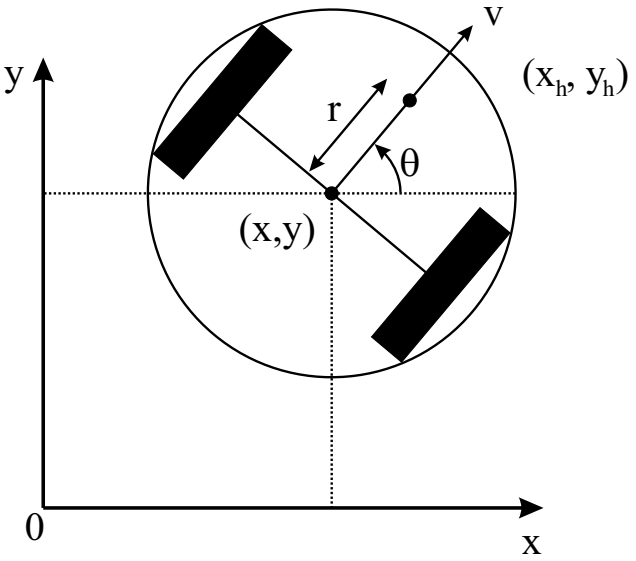

Fig. 8: Coordinates of the differential drive robot.

identical when communicating with different robots. In this case, we set $h=0.5 \mathrm{~s}$ and use the inherent delay as the uncertainty term $\Delta h_{i} \in[0.15 s, 0.195 s], \forall i=1,2,3,4$.

\section{B. Linearisation-Based Kinematic Model of E-Puck Robot}

Let $(x, y), \theta, v$ and $\omega$ denote the Cartesian positions, the orientation, the linear and the angular velocity of the robot. The kinematic model of the E-puck robot is described as

$$
\left[\begin{array}{l}
\dot{x} \\
\dot{y} \\
\dot{\theta}
\end{array}\right]=\left[\begin{array}{cc}
\cos \theta & 0 \\
\sin \theta & 0 \\
0 & 1
\end{array}\right]\left[\begin{array}{c}
v \\
\omega
\end{array}\right] .
$$

Fig. 8 describes the position of the robot in the global inertial frame. The robot is located at $P$ in the frame which is represented by $p=\left[\begin{array}{ll}x, y\end{array}\right]^{T}$. Consider the nonholonomic kinematic constraint, the head position $\hat{p}=\left[\begin{array}{ll}x_{h}, & y_{h}\end{array}\right]^{T}$ as shown in Fig. 8 is used to replace the inertial position. It can be verified that

$$
\left[\begin{array}{c}
\dot{x}_{h} \\
\dot{y}_{h} \\
\dot{\theta}
\end{array}\right]=\left[\begin{array}{cc}
\cos \theta & -r \sin \theta \\
\sin \theta & r \cos \theta \\
0 & 1
\end{array}\right]\left[\begin{array}{c}
v \\
\omega
\end{array}\right],
$$

where $r$ is the distance between the head position and the inertial position. By letting $u=\left[u_{x}, u_{y}\right]^{T}$ and $\eta=$ $[v, \omega]^{T}$, the linearisation-based kinematic model can be developed as $\eta(t)=H u(t)$ and $u(t)=\dot{\hat{p}}(t)$ with $H=$ $\left[\begin{array}{cc}\cos \theta & \sin \theta \\ -\frac{1}{r} \sin \theta & \frac{1}{r} \cos \theta\end{array}\right]$. Note that in the following experiments we choose $r=2.5 \mathrm{~cm}$.

\section{Static Formation with four E-puck robots}

The aim of this experiment is to show that the proposed control law will drive the robots to form a formation whose pattern is described as

$$
\mathcal{F}_{i}=\left[\begin{array}{c}
R \cos \left(\frac{2 \pi(i-1)}{N}+\psi\right) \\
R \sin \left(\frac{2 \pi(i-1)}{N}+\psi\right)
\end{array}\right], i=1,2,3,4,
$$

where $R$ is the distance between the centre of the formation frame and the robot, $\psi$ is the desired angle between $x$-axis and the first robot with respect to the centre of the formation. In this test, we choose $R=30 \mathrm{~cm}$ and $\psi=(\pi / 4) \mathrm{rad}$. 


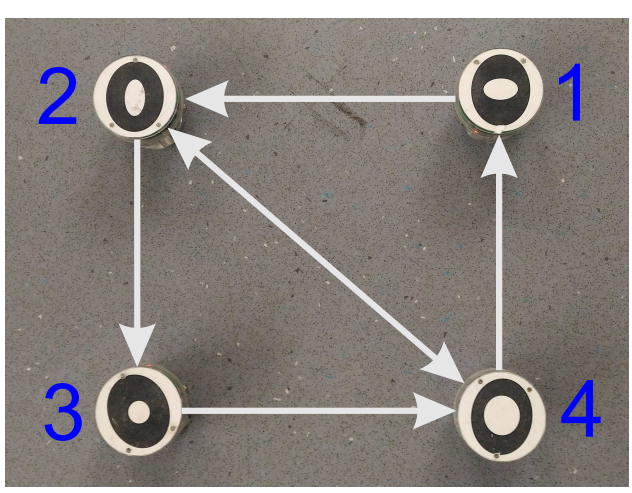

Fig. 9: Directed communication topology.

Suppose the directed graph in Fig. 9 is used to model the information exchange among robots. Accordingly, the Laplacian matrix $\mathcal{L}$ is given by

$$
\mathcal{L}=\left[\begin{array}{cccc}
1 & 0 & 0 & -1 \\
-1 & 2 & 0 & -1 \\
0 & -1 & 1 & 0 \\
0 & -1 & -1 & 2
\end{array}\right]
$$

and

$$
\hat{\mathcal{L}}=\left[\begin{array}{cccc}
1 & -0.5 & 0 & -0.5 \\
-0.5 & 2 & -0.5 & -1 \\
0 & -0.5 & 1 & -0.5 \\
-0.5 & -1 & -0.5 & 2
\end{array}\right]
$$

The eigenvalues of the Laplacian matrix $\hat{\mathcal{L}}$ are $\{0,1.0,2.0,3.0\}$, and zero is a simple eigenvalue. By setting $\alpha=\beta=0.5, m=19$ and $n=21$ for each protocol, the estimated upper bound of the settling time for protocol (11) is $34.5951 \mathrm{~s}$.

The experimental results of this scenario are displayed in Figs. 10, 11, and 12. Fig. 10 shows the formation achieving process at $t=0 \mathrm{~s}, 6 \mathrm{~s}, 12 \mathrm{~s}, 18 \mathrm{~s}, 24 \mathrm{~s}, 30 \mathrm{~s}$. In more detail, Fig. 11 visualizes the trajectories of robots from the initial poisitons represented by black dots. The red triangles representing the position of robots at time $t=10$ s show that the robots are in the process of forming the rectangle formation, while the blue squares represent the final position of robots. Furthermore, the final positions are reached before $t=20 \mathrm{~s}$ as seen in Fig. 12. It shows the robustness of the estimated formation settling time.
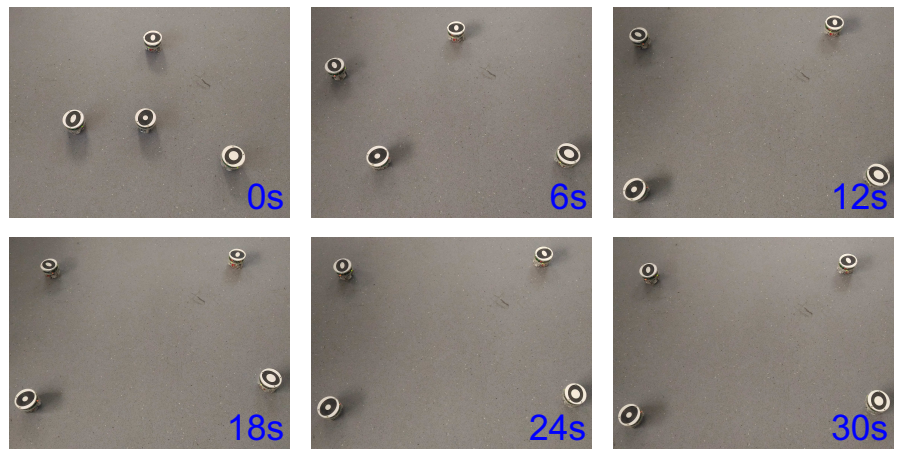

Fig. 10: Static formation with four robots.

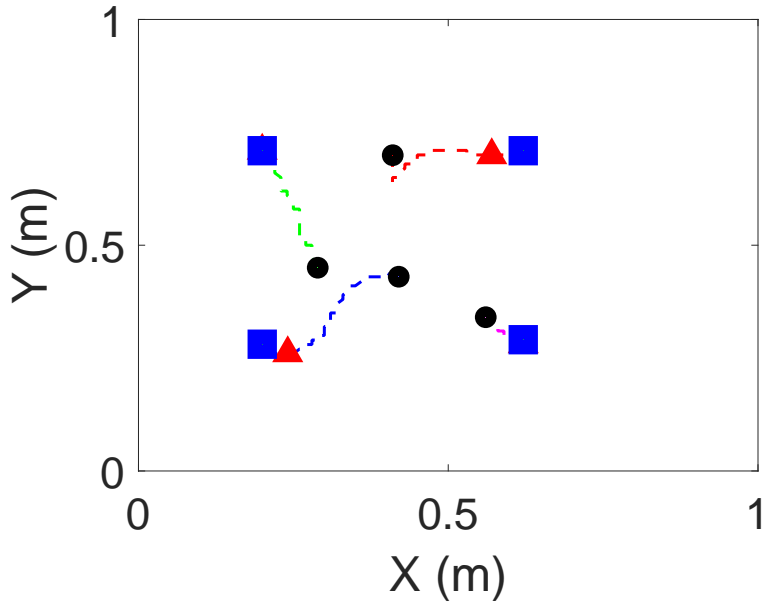

Fig. 11: Positions of each robots while forming a rectangle formation.
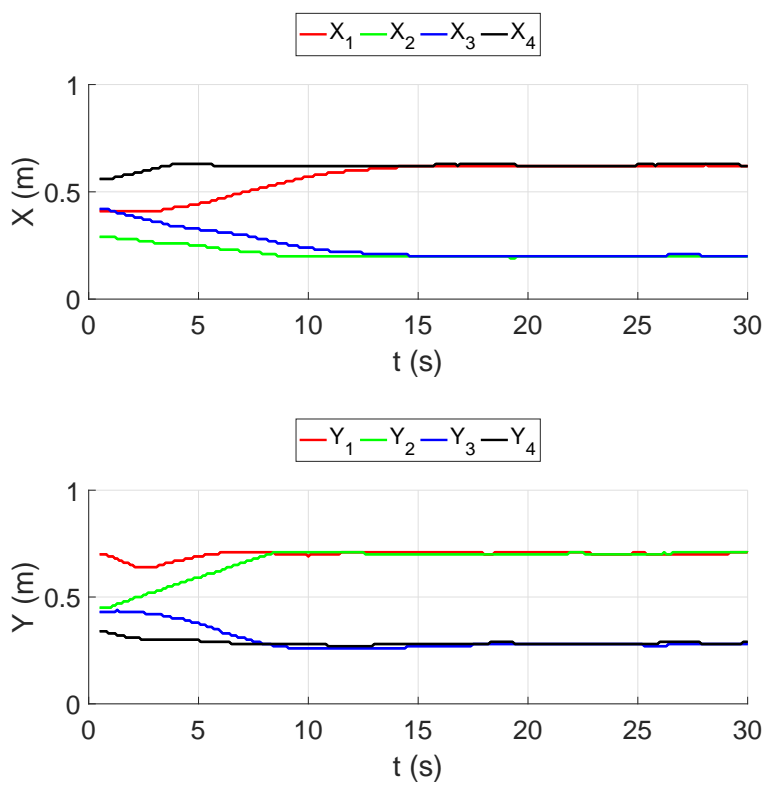

Fig. 12: Trajectories of the states with respect to time.

Remark 5: Notice that saturations of linear and angular velocities have to be enforced in the experiment due to the physical limitations of the robots. Compared with the simulation setting in Section IV, bigger value of $m / n$ is chosen in the test to avoid possible saturation. Furthermore, the approximation method introduced in [38] is used to deal with the implementation of the integral term in (3).

\section{Time-varying Formation with four E-puck robots}

For time-varying formations, four robots are commanded to move on the $x-y$ plane to form a time-varying formation in fixed-time. The formation is defined by

$$
\mathcal{F}_{i}(t)=\left[\begin{array}{c}
R \cos \left(\frac{2 \pi t}{T}+\frac{2 \pi(i-1)}{N}+\psi\right) \\
R \sin \left(\frac{2 \pi t}{T}+\frac{2 \pi(i-1)}{N}+\psi\right)
\end{array}\right], i=1,2,3,4,
$$

which represents the periodic time-varying formation and rotating around the formation centre with period $T$. In this 
case, we choose $R=30 \mathrm{~cm}$ and $\psi=0 \mathrm{rad}$. By doing a simple mathematical analysis, if the maximum velocity of robot is $5 \mathrm{~cm} / \mathrm{s}$, the value of $T$ must be larger or equal to $25.1327 \mathrm{~s}$ in order to make the robots follow the desired path. In this case, $T$ is chosen to be $50.2655 \mathrm{~s}$. The directed graph in Fig. 8 is used to model the information exchange among robots. By setting $\alpha=\beta=1, m=19$ and $n=21$ for each protocol, the estimated upper bound of the settling time for protocol (11) is $34.5951 \mathrm{~s}$.
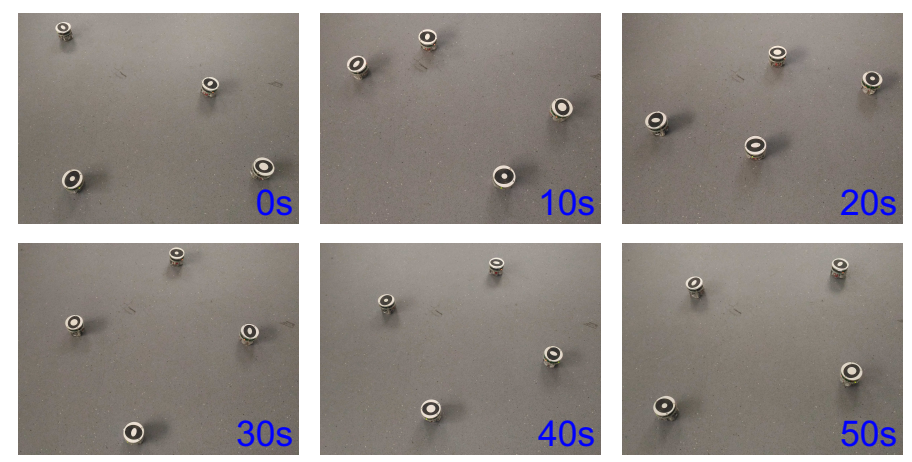

Fig. 13: Time-varying formation with four robots.

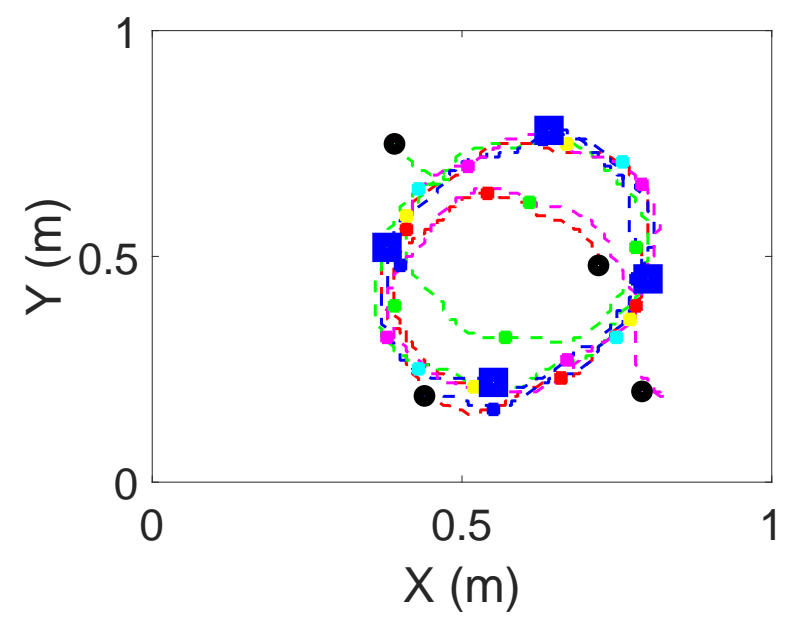

Fig. 14: Robots rotating around the centre of formation.

Figs. 13, 14 and 15 depict the experimental results of the proposed time-varying formation. In Fig. 13, we can see that the robots are initially deployed in random positions within the workspace. After 20 seconds, the robots rotate around the centre of formation. Fig. 14 shows the trajectories of robots starting from the initial positions as pointed by black dots. The red, green, blue, yellow, cyan and magenta dots indicate the positions of robots at different time instants (10s, 20s, 30s, etc.). The robots keep rotating around the centre of formation during the experiment, and then stop at some positions pointed by blue squares at the end time. In Fig. 15, the trajectories of robots with respect to time start to form sinusoidal waves before $\mathrm{t}=30 \mathrm{~s}$.

\section{Vi. Conclusion and Future Research}

This paper has investigated the impact of non-identical input delay in fixed-time formation control for multi-robot
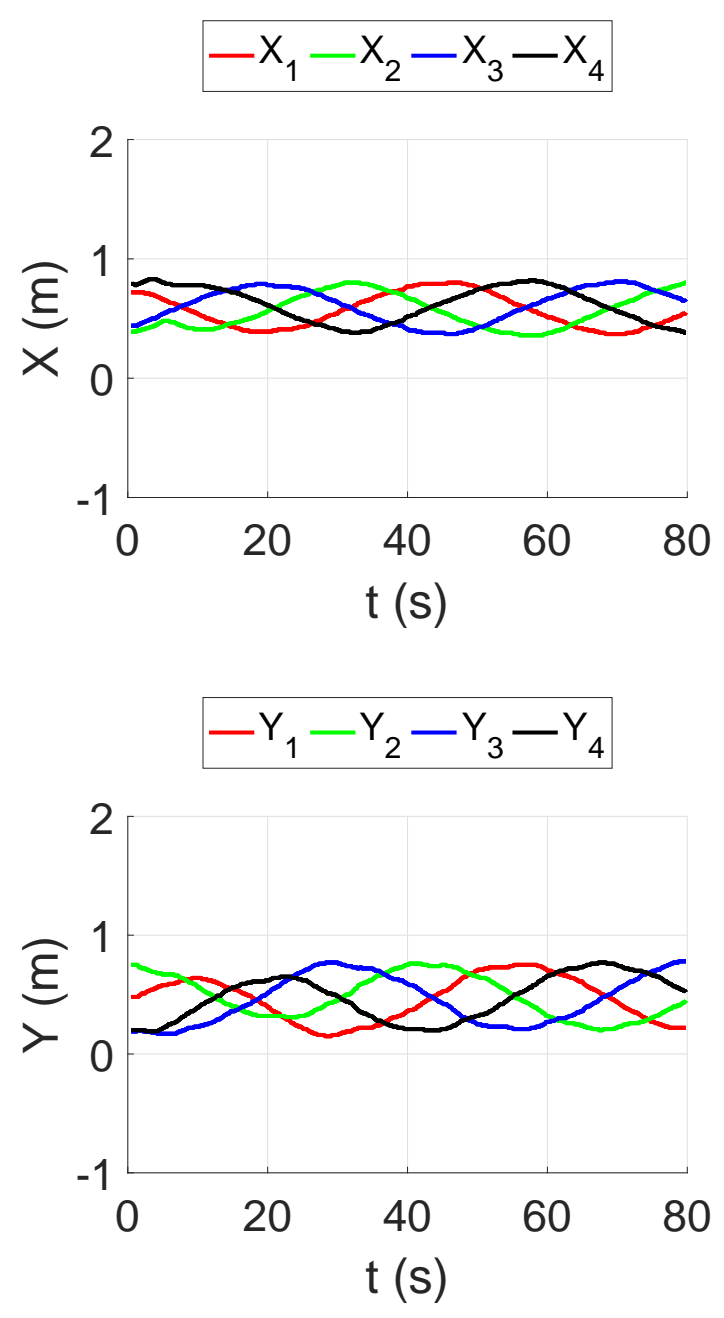

Fig. 15: Trajectories of the states with respect to time.

systems. This input delay may represent the communication delay and the processing and connecting time for the packets arriving at each agent. Based on the predictor strategy, a state transformation is applied for each agent and the extra terms remain in the transformed systems are carefully considered. A new class of fixed-time nonlinear formation protocols are constructed and a complete stability analysis is presented in a systematic framework of Lyapunov functionals. The upper bound of the formation settling time is independent of the system initial conditions such that the convergence time can be designed or estimated off-line. Finally, both simulation and experimental results are presented to show the effectiveness of the protocols proposed in this paper. Future work may focus on reducing the unnecessary information exchange and solving the oscillating problem for the robots in motion. Furthermore, the extension of the proposed results to the case involving a directed spanning tree is also listed in our future work. 


\section{REFERENCES}

[1] J. Fax and R. Murray, "Information flow and cooperative control of vehicle formation," IEEE Transactions on Automatic Control, vol. 49 no. 9, pp. 1465-1476, 2004.

[2] W. Ren, "Information consensus in multi-vehicle cooperative control," IEEE Control Systems Magazine, vol. 27, no. 2, pp. 71-82, 2007.

[3] R. Olfati-Saber and R. Murray, "Consensus problems in networks of agents with switching topology and time-delays," IEEE Transactions on Automatic Control, vol. 49, no. 9, pp. 1520-1533, 2004.

[4] W. Ren and R. Beard, "Consensus seeking in multiagent systems under dynamically changing interaction topologies," IEEE Transactions on Automatic Control, vol. 50, no. 5, pp. 655-661, 2005.

[5] W. Yu, G. Chen, J. Lu, and J. Kurths, "Synchronization via pinning control on general complex networks," SIAM Journal on Control and Optimization, vol. 51, no. 2, pp. 1395-1416, 2013.

[6] Z. Li, W. Ren, X. Liu, and M. Fu, "Consensus of multi-agent systems with general linear and Lipschitz nonlinear dynamics using distributed adaptive protocols," IEEE Transactions on Automatic Control, vol. 58, no. 7, pp. 1786-1791, 2013.

[7] J. Wang and M. Xin, "Integrated optimal formation control of multiple unmanned aerial vehicles," IEEE Transactions on Control Systems Technology, vol. 21, no. 5, pp. 1731-1744, 2013.

[8] A. Karimoddini, H Lin, B. M. Chen, and T. H. Lee, "Hybrid threedimensional formation control for unmanned helicopters," Automatica, vol. 49, no. 2, pp. 424-433, 2013.

[9] Z. Ding and Z. Li, "Distributed adaptive consensus control of nonlinear output-feedback systems on directed graphs," Automatica, vol. 72, pp. 46-52, 2016.

[10] H. T. Zhang, Z. Chen, and M. C. Fan, "Collaborative control of multivehicle systems in diverse motion patterns," IEEE Transactions on Control Systems Technology, vol. 24, no. 4, pp. 1488-1494, 2016.

[11] B. Wang, J. Wang, B. Zhang, and X. Li, "Global cooperative control framework for multiagent systems subject to actuator saturation with industrial applications," IEEE Transactions on Systems, Man, and Cybernetics: Systems. In press.

[12] X. Dong, B. Yu, Z. Shi, and Y. Zhong, "Time-varying formation control for unmanned aerial vehicles: Theories and applications," IEEE Transactions on Control Systems Technology, vol. 23, no. 1, pp. 340348, 2015.

[13] Z. Ding, "Distributed adaptive consensus output regulation of networkconnected heterogeneous unknown linear systems on directed graphs," IEEE Transactions on Automatic Control, vol. 62, no. 9, pp. 4683-4690, 2017.

[14] X. Dong, Y. Zhou, Z. Ren, and Y. Zhong, "Time-varying formation tracking for second-order multi-agent systems subjected to switching topologies with application to quadrotor formation flying," IEEE Transactions on Industrial Electronics, vol. 64, no. 6, pp. 5014-5024, 2017.

[15] M. Macktoobian and M. Aliyari Sh, "Optimal distributed interconnectivity of multi-robot systems by spatially-constrained clustering," Adaptive Behavior, vol. 25, no. 2, 96-113, 2017.

[16] W. Xu and D. W. C. Ho, "Clustered event-triggered consensus analysis: An impulsive framework," IEEE Transactions on Industrial Electronics, vol. 63, no. 11, pp. 7133-7143, 2016.

[17] W. Xu, Z. Wang, and D. W. C. Ho, "Finite-horizon $H_{\infty}$ consensus for multiagent systems with redundant channels via an observer-type eventtriggered scheme," IEEE Transactions on Cybernetics, vol. 48, no. 5, pp. 1567-1576, 2018.

[18] Y. Mei, Y. H. Lu, Y. C. Hu, and C. G. Lee, "Deployment of mobile robots with energy and timing constraints," IEEE Transactions on Robotics, vol. 22, no. 3, pp. 507-522, 2006.

[19] S. P. Bhat and D. S. Bernstein, "Finite-time stability of continuous autonomous systems," SIAM Journal of Control and Optimization, vol. 38, no. 3, pp. 751-766, 2000.

[20] F. Xiao, L. Wang, J. Chen, and Y. Gao, "Finite-time formation control for multi-agent systems," Automatica, vol. 45, no. 11, pp. 2605-2611, 2009.

[21] S. Khoo, L. Xie, and Z. Man, "Robust finite-time consensus tracking algorithm for multirobot systems," IEEE/ASME transactions on mechatronics, vol. 14, no. 2, pp. 219-228, 2009.

[22] Y. Zhao, Z. Duan, G. Wen, and Y. Zhang, "Distributed finite-time tracking control for multi-agent systems: An observer-based approach," Systems \& Control Letters, vol. 62, no. 1, pp. 22-28, 2013.

[23] M. Ou, H. Du, and S. Li, "Finite-time formation control of multiple nonholonomic mobile robots," International Journal of Robust and Nonlinear Control, vol. 24, no. 1, pp. 140-165, 2014
[24] H. Du, S. Li, and X. Lin, "Finite-time formation control of multiagent systems via dynamic output feedback," International Journal of Robust and Nonlinear Control, vol. 23, no. 14, pp. 1609-1628, 2013.

[25] Z. Zuo and L. Tie, "A new class of finite-time nonlinear consensus protocols for multi-agent systems," International Journal of Control, vol. 87 , no. 2, pp. 363-370, 2014.

[26] Z. Zuo and L. Tie, "Distributed robust finite-time nonlinear consensus protocols for multi-agent systems," International Journal of Systems Science, vol. 47, no. 6, pp. 1366-1375, 2016.

[27] Z. Zuo, "Nonsingular fixed-time consensus tracking for second-order multi-agent networks," Automatica, vol. 54, pp. 305-309, 2015.

[28] X. He, Z. Wang, and D. H. Zhou, "Robust fault detection for networked systems with communication delay and data missing," Automatica, vol. 54, no.11, pp. 2634-2639, 2009.

[29] W. Qiao and R. Sipahi, "Consensus control under communication delay in a three-robot system: Design and experiments," IEEE Transactions on Control Systems Technology, vol. 24, no. 2, pp. 687-694, 2016.

[30] A. Abdessameud and A. Tayebi, "Formation control of VTOL unmanned aerial vehicles with communication delays," Automatica, vol. 47, no. 11, pp. 2383-2394, 2011.

[31] X. Dong, J. Xi, G. Lu, and Y. Zhong, "Formation control for highorder linear time-invariant multiagent systems with time delays," IEEE Transactions on Control of Network Systems, vol. 1, no. 3, pp. 232-240, 2014.

[32] Z. Artstein, "Linear systems with delayed controls: a reduction," IEEE Transactions on Automatic Control, vol. 27, no. 4, pp. 869-879, 1982.

[33] C. Wang, Z. Zuo, Z. Lin, and Z. Ding, "Consensus control of a class of Lipschitz nonlinear systems with input delay," IEEE Transactions on Circuits and Systems I: Regular Papers, vol. 62, no. 11, pp. 2730-2738, 2015.

[34] Z. Zuo, C. Wang, and Z. Ding, "Robust consensus control of uncertain multi-gent systems with input delay: a model reduction method," International Journal of Robust and Nonlinear Control, vol. 27, no. 11, pp. 1874-1894, 2017.

[35] Z. Zuo, Q.-L. Han, B. Ning, X. Ge, X.-M. Zhang, "An overview of recent advances in fixed-time cooperative control of multi-agent systems," IEEE Trans. Ind. Inform., vol. 14, no. 6, pp. 2322-2334, 2018.

[36] Z. Zuo, W. Yang, L. Tie, and D. Meng, "Fixed-time consensus for multiagent systems under directed and switching interaction topology," In: Proc. 2014 American Control Conf., Portland, OR, USA, June 2014, pp. $5133-5138$

[37] T. Krajník, M. Nitsche, J. Faigl, P. Vaněk, M. Saska, L. Přeučil, T. Duckett, M. Mejail, “A practical multirobot localization system,” Journal of Intelligent \& Robotic Systems, vol. 76, no. 3-4, pp. 539-562, 2014.

[38] Q. C. Zhong, Robust control of time-delay systems. Springer Science \& Business Media, 2006. 